\title{
Evaluating the Use of Pictograms for the Improvement in Patient Understanding of Medication Instruction-A Pilot Study
}

\author{
Dominique Hanslo ${ }^{1}$, Velisha Ann Perumal-Pillay ${ }^{1} \&$ Fatima Suleman ${ }^{1}$ \\ ${ }^{1}$ Discipline of Pharmaceutical Sciences, School of Health Sciences, University of KwaZulu-Natal, Durban, 4000, \\ South Africa \\ Correspondence: Velisha Perumal-Pillay, Discipline of Pharmaceutical Sciences, School of Health Sciences, \\ University of KwaZulu-Natal, Durban, 4000, South Africa. Tel: 27-31-260-7766. E-mail: perumalv@ukzn.ac.za
}

Received: August 29, 2019 Accepted: November 11, 2019 Online Published: November 13, 2019

doi:10.5539/gjhs.v11n13p91 URL: https://doi.org/10.5539/gjhs.v11n13p91

\begin{abstract}
The first step in ensuring patient compliance is to ensure that the patient understands how to use their medication. The aim of the study was to determine if patients understanding of medication use can be improved by using pictograms. A simple randomized control test was set up whereby 50 patients were selected to participate, of which 25 people were included in the test group to receive medication labels based on pictograms as well as the usual counselling and 25 people were included in the control group and received the usual labelling of their medication and counselling. Questionnaires were used to determine the patient's demographic information and health literacy on the day of inclusion into the study, while another questionnaire was completed 3 to 7 days later to determine patient understanding of how to use their medication and if they used their medication correctly. At a $95 \%$ confidence interval and $\mathrm{p}<0.05$ there is a relationship between improved understanding and the use of pictograms. Pictograms improve patient's understanding of how to use their medication, which in turn lead to an improvement in adherence.
\end{abstract}

Keywords: pictograms, medication use, adherence, South Africa

\section{Introduction}

Medication plays a role in improving clinical outcomes, increasing life expectancy and improving quality of life. However for medication to be effective it has to be used correctly (Bunker, 2001; Compendium, 2005). This is where the pharmacist plays a vital role. Pharmacists not only provide patients with their medication, but also provide information on the safe and correct use of the medication, i.e. how and when to use (Bunker, 2001). Added to this pharmacists are also tasked with ensuring patient adherence to ensure a favourable health outcome (Compendium, 2005). Patients' adherence depends partly on their ability to understand and interpret the instructions given by the pharmacist (FIP, 2014; Leong, Tam, Xu, \& Peters, 2018). With this in mind, all patients are given medication instructions written on a label and then supplemented with counselling i.e. telling the patient how, when and where to use the medication. However, barriers to communication hinder the ability of the patient to correctly interpret the information and thereby using the medication correctly (Cleary, 1999; FIP, 2014). This in turn leads to a decrease in compliance and further to a decline in successful treatment (Conradie, 2009; Osterberg $\&$ Blaschke, 2005). With this in mind a study was devised using a simple control test to determine if a patient's understanding of drug use can be improved by using pictograms. A secondary objective was to determine if this understanding of the medicine use would also lead to an improvement in adherence.

Language barriers are huge problems in South Africa as not only are there eleven official languages, but with people migrating from all over the world the number of languages spoken in South Africa is far more than this ("South Africa," 2012; StatsSA, 2011). Although English is spoken by more people and regarded as the international language, according to Statistics South Africa in 2001 only $8.2 \%$ of South Africans use English as a home language (Erasmus-Kritzinger, Swart, \& Mona, 2000; Larry, 2002; "South Africa," 2012; StatsSA, 2001). Very often the understanding of English is basic and limited, making word choice important when communicating (Cleary, 1999). The simpler the words used the more likely the message will be interpreted correctly e.g. using ask instead of query (Cleary, 1999; Larry, 2002). However, even when a common language is spoken there is no guarantee that the message send will be correctly received by the receiver. Depending on one's frame of reference, words can have different meanings (Cleary, 1999). To complicate matters even further, in 2001 the percentage of 
persons with no schooling was $17.9 \%$ and according to the National Institute for the Deaf about four-million South Africans have a hearing difficulty, while a further 1.5-million are classified as "profoundly deaf" ("South Africa," 2012).

Another important aspect of communication is culture (Cleary, 1999). South Africa has a large and diverse population with many different cultures (Cleary, 1999; "South Africa," 2012; StatsSA, 2011). A complication to culture is the term subcultures, where -within a particular culture group- there can be further division based on profession, ethnicity, religion, demographics and language ("South Africa," 2012; StatsSA, 2001, 2011). The greater the cultural differences the greater the communication gap (Cleary, 1999; FIP, 2014). Pictograms rely on patients being able to understand pictures, however one element of culture is symbols which can have vastly different meanings depending on the culture for instance in western culture white is a sign of purity but in eastern cultures it is a sign of mourning (Cleary, 1999; FIP, 2014). The cultural experiences can and is often at odds with each other.

Adherence relies on patients understanding of how to use the medication and then while unobserved, to use the medication correctly (Osterberg \& Blaschke, 2005). Non-adherence can be seen as missing any doses, decreasing dosing intervals, delays in filling prescriptions or not filling prescriptions (Huber, 2013; Osterberg \& Blaschke, 2005; Steel, Nwokike, \& Joshi, 2007). Non-adherence can lead to increased health care, reduction in productivity, loss of medication, increased morbidity and/or mortality (Horne, 2006; Huber, 2013; Osterberg \& Blaschke, 2005; Steel et al., 2007). However, although giving clear understandable instructions on how and when to use medication helps with adherence, it does not guarantee adherence (Horne, 2006; Mansoor \& Dowse, 2006; Osterberg \& Blaschke, 2005). This is because the person required to take the medication may not see the same necessity or urgency as the healthcare professional. Several reasons for non-compliance have been suggested ranging from complexity of regimen, cost, side effects, route of administration and physiological factors such as denial(Horne, 2006; Osterberg \& Blaschke, 2005). Patients can also be unintentionally non-adherent when they fail to understand the instructions given. It is estimated that between $5-10 \%$ of people are unable to recall the information given to them in the consultation room and generally patients are more compliant on acute medication for short periods than chronic medication (Horne, 2006; Huber, 2013; Osterberg \& Blaschke, 2005; Steel et al., 2007).

Even when there is no language or cultural barriers, patients are expected to remember the instructions given by the pharmacist and only have the written instruction on the label as reference (Cleary, 1999; Conradie, 2009; FIP, 2014; Larry L. Barker, 2002). There are two problems with labels, firstly the label instructions assumes that the patient is literate, and secondly that the language on the label is easy to understand(FIP, 2014). Patients often become confused on how to correctly use the medication and this leads to non-adherence (Cleary, 1999; FIP, 2014; Larry L. Barker, 2002). Pictograms were developed by the International Pharmaceutical Federation (FIP) in an attempt to assist pharmacists in improving communication when there is a language barrier (FIP, 2014). The aim was to provide information in the form of pictures. The pictogram does not take into consideration the language spoken by the patient but rather the culture of the patient. Black and white is the only colours used and the only information depicted by the pictograms is the dosage, frequency, precautions and indications (FIP, 2014).

\section{Methods}

The study was approved by the University of KwaZulu-Natal Biomedical Research Ethics Committee (BE343/13). The sample was selected from a Health Centre in the Tygerberg District in the Western Cape in South Africa in which the population group was predominately coloured, Afrikaans speaking.

Patients for possible inclusion were identified by an interpreter at the reception of the facility on the basis that they were unable to speak English or Afrikaans as a first language. The interpreter also assisted with the administration of the questionnaires as well as the translation of the consent forms. The inclusion criteria were as follows: (a) first language should be one of the official languages excluding English and Afrikaans; (b) male or female between 18 and 65 years old; (c) first time using the prescribed medication. Exclusion criteria were as follows: (a) ability to speak English and/or Afrikaans; (b) has an interpreter or caregiver; (c) previously used the prescribed medication.

After obtaining informed consent from participants, a simple randomised controlled test was set up with the control group receiving the normal labelling of medication and information about drug usage and the test group received labelling of their medication in the form of a pictogram (Ros \& Martina, 2001). Both groups received the same information with the only difference being that the test group received pictograms to aid in the understanding of how to use the medication.

A questionnaire was used to test both groups understanding of how the medication should be used and how they used their medication. The questionnaire was divided into three parts. The first part of the questionnaire revolved 
around the demographics of the participants such as age, home language as well as the type of medication received. The second part was a short literacy test to establish the health literacy of the patient (according to Rapid Estimate of Adult Literacy in Medicine - Short Form (REALM-SF)). For the third part of the questionnaire participants were contacted via telephone approximately 3-7 days after they started using their medication to determine how well they understood the instructions on how to take their medication and to determine adherence by doing pill counts. The participant was asked to do the pill count while on the telephone. The total number of doses taken compared to the total number of doses that should have been taken from the time of initiation of treatment was used to determine the percentage adherence. The time frame for the questionnaires and interview was approximately 20-30 minutes in total per participant. The interviews were not recorded and all information given by the patients was written on the questionnaire.

Both qualitative and quantitative analyses were used to draw results and conclusions. The results were statistically analysed using the Chi-square test to determine if there was an improvement in understanding of medication use with the aid of pictogram and subsequently if this improvement led to improved compliance. The t-test was used to compare the baseline characteristics of both the test group and control group to determine that the population means were equal. A $95 \%$ confidence interval was used to calculate the outcomes.

\section{Results}

Fifty participants were recruited from the period 1 August -9 October 2014. The results of the study are presented below.

Table 1. Demographic characteristics of participants

\begin{tabular}{|c|c|c|c|}
\hline \multirow{2}{*}{ Demographic details } & & \multirow{2}{*}{$\begin{array}{l}\text { Control group } \\
\mathrm{n}=24\end{array}$} & \multirow{2}{*}{$\begin{array}{l}\text { Test group } \\
\mathbf{n}=\mathbf{2 3}\end{array}$} \\
\hline & & & \\
\hline \multirow{2}{*}{ Gender } & Female & $15(62.5)$ & $14(60.9)$ \\
\hline & Male & $9(37.5)$ & $9(39.1)$ \\
\hline \multirow{3}{*}{ Age(years) } & $18-29$ & $6(25)$ & $6(26.1)$ \\
\hline & $30-40$ & $15(62.5)$ & $14(60.9)$ \\
\hline & $>40$ & $3(12.5)$ & $3(13.0)$ \\
\hline \multirow{3}{*}{ Highest Qualifications according to REALM-SF } & 4-6th grade & $8(33.3)$ & $7(30.4)$ \\
\hline & $7-8^{\text {th }}$ grade & $13(54.2)$ & $13(56.5)$ \\
\hline & $\geq 9^{\text {th }}$ grade & $3(12.5)$ & $3(13.0)$ \\
\hline \multirow{2}{*}{ Ethnicity } & African & $21(87.5)$ & $21(91.3)$ \\
\hline & Coloured & $3(12.5)$ & $2(8.6)$ \\
\hline \multirow{3}{*}{ Marital Status } & Single & $9(37.5)$ & $12(52.1)$ \\
\hline & Married & $15(62.5)$ & $9(39.1)$ \\
\hline & Divorced & $0(0)$ & $2(8.7)$ \\
\hline
\end{tabular}

There was no significant difference in the patients' demographic characteristics between the two groups. 
Table 2. Participants' responses to questions related to their understanding of how to use their medicines

\begin{tabular}{llc}
\hline Questions & Control group & Test group \\
& $\mathbf{n = 2 4}$ & $\mathbf{n}=\mathbf{2 3}$ \\
\hline 1.Provide information on the dosage of your medicines & $18(75.0)$ & $18(91.3$ \\
2. Provide information on directions for use & $18(75.0)$ & $15(65.2)$ \\
3. Provide information on side effects of your medicines & $8(33.3)$ & $11(47.8)$ \\
4. What instructions were you given on how to use the medicines? & $8(33.3)$ & $12(52.2)$ \\
5. Explain the instructions on the label/pictogram & $10(41.7)$ & $17(73.9)$ \\
6 Explain in your own words how you took the medication & $14(58.3)$ & $14(60.9)$ \\
7. How much medication do you have left? & $9(37.5)$ & $16(69.6)$ \\
\hline
\end{tabular}

Patients understanding of the dosage were high for both groups; however side effects do not seem to be well understood by patients in both the control and test group. This could be because the term is unfamiliar or due to recall bias. Directions for use was better understood by the control group. When participants were requested to explain how they took their medicines, there was a poor response in the control group only scoring $33.3 \%$ and the test group scoring $60.9 \%$. This could be attributed to the pictograms assisting them in recall.

Using the answers to the question which investigated patient's understanding of the instruction provided on the label or pictogram, the null hypothesis was determined to establish if there is an increased understanding in medication use with using pictograms. The results showed that there was a $95 \%$ confidence level that using pictogram improves patients understanding of medication use.

The null hypothesis was determined to establish if there was an increase in compliance with understanding of medication use when using pictograms by using the answers to the question: How much medicines do you have left? Using a $95 \%$ confidence interval and $\mathrm{p}<0.05$, it was established that with an increased understanding of medication use there was an increase in adherence.

Table 3. The effect of age on understanding the label or pictogram

\begin{tabular}{lll}
\hline Age (years) & Control group $\mathbf{n = 2 4}$ & Test group $\mathbf{n = 2 3}$ \\
\hline $18-29(\mathrm{n}=7)$ & $4(57.1)$ & $5(71.4)$ \\
$30-40(\mathrm{n}=13)$ & $5(38.5)$ & $11(84.6)$ \\
$>40(\mathrm{n}=3)$ & $1(33.3)$ & $1(33.3)$ \\
\hline
\end{tabular}

At a $\mathrm{p}<0.025$ there is a significant increase in understanding with age for the test group.

Table 4. The effect of gender on understanding the label or pictogram

\begin{tabular}{lll}
\hline Gender & Control group $\mathbf{n = 2 4}$ & Test group $\mathbf{n = 2 3}$ \\
\hline female $(\mathrm{n}=14)$ & $6(42.9)$ & $12(85.7)$ \\
male $(\mathrm{n}=9)$ & $4(44.4)$ & $5(55.6)$ \\
\hline
\end{tabular}

At a $95 \%$ confidence interval there is no increase in understanding with gender for both groups. 
Table 5. Qualitative analysis of reasons patients did not take their medication

\begin{tabular}{lll}
\hline Reason & $\begin{array}{l}\text { Control group } \\
\mathbf{n = 1 5}\end{array}$ & $\begin{array}{l}\text { Test group } \\
\mathbf{n}=\mathbf{7}\end{array}$ \\
\hline Forgot to take medication & $8(53.3)$ & $5(71.4)$ \\
Felt better & $4(26.7)$ & $2(28.6)$ \\
Didn't need the medication & $2(13.3)$ & $0(0)$ \\
No reason & $1(6.7)$ & $0(0)$ \\
\hline
\end{tabular}

Forgetfulness seemed to be the main reason for both the control group $(53.3 \%)$ and the test group $(71.4 \%)$ when they did not take their medication.

\section{Discussion}

Although the sample size was relatively small (50 participants only), it took just over 2 months to get enough patients to complete the study. Recruiting patients that fit the criteria for inclusion in the study was difficult as most of the patients at the Health Centre spoke English or Afrikaans as a first language. The next difficulty was finding patients that were taking the medication for the first time.

Three patients were lost to follow up, which was surprisingly positive because of the transient nature of people coming into the area it was expected that more patients would be lost to follow up. However, technology in the form of cell phones assisted in ensuring that most people were reachable.

Patients understanding of side effects were poor for both groups. There seemed to be a lack of patient's education with respect to their understanding of what side effects are. This needs to be addressed during counselling and maybe because the interpreter was not medically trained they also lacked the ability to sufficiently probe participants.

According to the results the average age was 34 with ages ranging from 22 to 62 , however only two participants were 62 years old. Most patients were between 28-39 years old. A larger participant pool with more age variation is required to determine if age and understanding is really of significance.

This study is similar to the study done by (Yin et al., 2008) who evaluated the efficacy of a pictogram based health literacy intervention to reduce medication administration errors by caregivers of young children, in that one group received the standard treatment while a second group received the addition of pictograms. The results of their study confirm that patients understanding of medication use are enhanced with pictograms. Our study has the same outcome as just over $50 \%$ of patients in the test group could recall information they were given but this increased to $73.9 \%$ when a pictogram was added which meant that the pictograms enhanced their understanding. For both the control and the test group their understanding of the label/pictogram directly influenced that pill count. For the control group $41.7 \%$ understood the label and had an adherence of $37.5 \%$ while the test group had a $73.9 \%$ understanding of the pictograms which translated into a $69.6 \%$ adherence level. Dowse and Ehlers (Ros Dowse \& Martina S Ehlers, 2001) determined that locally produced pictograms were more acceptable to enhancing patients understanding and these pictograms were used in our study to determine if there was an increased understanding of how to use the medication with pictograms.

Unlike the study conducted by Mansoor and Dowse (Mansoor \& Dowse, 2006) which focused on isiXhosa patients from the Eastern Cape, our study was conducted in the Western Cape with patients speaking both isiXhosa and IsiZulu. However the results still showed that pictograms enhanced patients understanding of medication use. Although our study did not focus on the reasons for non-adherence, it seems that the major reason for not taking the medication seem to be forgetfulness. This could be because patients felt well after a couple of days and did not feel the need or urgency to continue taking their medication.

Other studies that showed pictograms improved patient understanding of medicine use include: a study conducted in Hong Kong by (Ng, Chan, \& Ho, 2017) which showed that pictograms improved comprehension of medication information for older people and that pictograms on labels better conveyed medication instructions; and a study by (Leong et al., 2018) in the United States to determine if pictograms enabled patients' ability to correctly fill a pill box showed that although the pictograms did not enhance patients' ability to do this, patients reported that pictograms helped them understand medicine instructions and felt pictograms should appear on all medicine labels. 
Our pilot study was successfully conducted in the Western Cape Province of South Africa and showed promising results on evaluating the use of pictograms for improved medicine use by patients. Healthcare policymakers for the province can use these results to advocate for and include a policy for the inclusion of pictograms for medicine use, especially for specific populations such as those with certain disbailities and hearing disorders who cannot benefit from the conventional, verbal method of instruction and counselling provided when medicines are dispensed. Furthermore, this study can be upscaled to include other provinces in the country to consolidate our results.

\section{Conclusions}

Pictograms have a place in enhancing counselling and serving as a reminder to patients on how to use their medication. With the improved understanding of how to use their medication, patients are more likely to be adherent to their medication regimens.

There seemed to be a lack of understanding of what constituted a side effect. This is an area that needs to be addressed and could well be a reason for non-adherence as patients may experience side effects but are not able to relate these to the health care professional. Patients' understanding of the labelling of their medication can be enhanced by using pictograms. However, the labelling should be in the patient's first language. The reasons for non-adherence was not the focus of the study, but the reasons for non-adherence may be able to assist in improving counselling and pictograms to ensure that patients are more adherent.

The area in which the study was conducted is predominately a coloured, Afrikaans speaking population. The study results may have been better results if it was conducted in an area where the majority of people don't speak English or Afrikaans as a first language. Although the pictograms were previously tested for being culturally acceptable to isiXhosa speaking patients, the pictograms were not tested to see if it was culturally acceptable to the people in the area of the Health Centre. Such studies, investigating cultural acceptance of pictograms is rare in South Africa.

\section{Acknowledgments}

Dr. V.A. Perumal-Pillay is a University of KwaZulu-Natal (UKZN) Developing Research Innovation, Localisation and Leadership in South Africa (DRILL) fellow. DRILL, is a NIH D43 grant (D43TW010131) awarded to UKZN in 2015 to support a research training and induction programme for early career academics. The content is solely the responsibility of the authors and does not necessarily represent the official views of DRILL and the National Institutes of Health.

\section{Competing Interests Statement}

The authors declare that there are no competing or potential conflicts of interest.

\section{References}

Bunker, J. P. (2001). The role of medical care in contributing to health improvements within societies. International Journal of Epidemiology, 30(6), 1260-1263. https://doi.org/10.1093/ije/30.6.1260

Cleary, S. (1999). Communication Handbook: Juta.

Compendium, P. P. L. (2005). Lexis-Nexis.

Conradie, C., Konig, T., Koti, S., Pillay, C., \& Valkhoff, J. (2009). Management Communication (2nd ed.). South Africa.

Erasmus-Kritzinger, L., Swart, M., \& Mona, V. (2000). Advanced communication Lynnwood Ridge: Afritech.

FIP. (2014). Pictogram project. Retrieved from https://www.fipfoundation.org/pictogram-project/

Horne, R. (2006). Compliance, adherence, and concordance: implications for asthma treatment. Chest, 130(1), 65S-72S. https://doi.org/10.1378/chest.130.1_suppl.65S

Huber, D. (2013). Leadership and nursing care management. Elsevier Health Sciences.

Larry L., \& Barker, D. R. G. (2002). Communication (8th ed.). Allyn and Bacon.

Leong, M., Tam, V., Xu, T., \& Peters, M. (2018). Understanding Medication Schedules: Do Pictograms Help? Journal of Patient Safety, 14(2), e19-e24. https://doi.org/10.1097/PTS.0000000000000241

Mansoor, L., \& Dowse, R. (2006). Written medicines information for South African HIV/AIDS patients: does it enhance understanding of co-trimoxazole therapy? Health Education Research, 22(1), 37-48. https://doi.org/10.1093/her/cyl039

Ng, A. W. Y., Chan, A. H. S., \& Ho, V. W. S. (2017). Comprehension by older people of medication information with or without supplementary pharmaceutical pictograms. Applied Ergonomics, 58, 167-175. 
https://doi.org/10.1016/j.apergo.2016.06.005

Osterberg, L., \& Blaschke, T. (2005). Adherence to Medication. New England Journal of Medicine, 353(5), 487-497. https://doi.org/10.1056/NEJMra050100

Ros, D., \& Martina, S. E. (2001). The evaluation of pharmaceutical pictograms in a low-literate South African $\begin{array}{lllll}\text { population. Patient Education } & \text { 8nd }\end{array}$ https://doi.org/10.1016/S0738-3991(00)00197-X

South Africa. (2012). Retrieved from https://www.south-africa-info.co.za/country

StatsSA. (2001). Census 2001. Retrieved from https://www.statssa.gov.za/publications/P0302/P03022011.pdf

StatsSA. (2011). Census 2011. Retrieved from http://www.statssa.gov.za

Steel, G., Nwokike, J., \& Joshi, M. P. (2007). Development of a multi-method tool to measure ART adherence in resource-constrained settings: the South Africa experience.

Yin, H. S., Dreyer, B. P., van Schaick, L., Foltin, G. L., Dinglas, C., \& Mendelsohn, A. L. (2008). Randomized Controlled Trial of a Pictogram-Based Intervention to Reduce Liquid Medication Dosing Errors and Improve Adherence Among Caregivers of Young Children. JAMA Pediatrics, 162(9), 814-822. https://doi.org/10.1001/archpedi.162.9.814

\section{Copyrights}

Copyright for this article is retained by the author(s), with first publication rights granted to the journal.

This is an open-access article distributed under the terms and conditions of the Creative Commons Attribution license (http://creativecommons.org/licenses/by/4.0/). 